\title{
Intranasal Esthesioneuroblastoma: CT Patterns Aid in Preventing Routine Nasal Polypectomy
}

\author{
(D) M.E. Peckham, (D) R.H. Wiggins III, (DR.R. Orlandi, (D) Y. Anzai, (D) W. Finke, and (D) H.R. Harnsberger
}

\begin{abstract}
BACKGROUND AND PURPOSE: Esthesioneuroblastoma is a neuroectodermal tumor that commonly arises in the nasal cavity olfactory recess and, when isolated to the intranasal cavity, can be indistinguishable from benign processes. Because lesional aggressiveness requires a more invasive operation for resection than polypectomy, patients with isolated intranasal lesions were studied to define distinguishing CT characteristics.

MATERIALS AND METHODS: Patients with intranasal esthesioneuroblastoma and controls without esthesioneuroblastoma with olfactory recess involvement were identified by using a report search tool. Studies demonstrating skull base invasion and/or intracranial extension were excluded. The imaging spectrum of these lesions was reviewed on both CT and MR imaging, and CT findings were compared with those of controls without esthesioneuroblastoma. Two blinded readers assessed subjects with esthesioneuroblastomas and controls without esthesioneuroblastoma and, using only CT criteria, rated their level of suspicion for esthesioneuroblastoma in each case.

RESULTS: Eight histologically proved cases of intranasal esthesioneuroblastoma were reviewed. All cases had CT demonstrating 3 main findings: 1) an intranasal polypoid lesion with its epicenter in a unilateral olfactory recess, 2) causing asymmetric olfactory recess widening, and 3) extending to the cribriform plate. Twelve patients with non-esthesioneuroblastoma diseases involving the olfactory recess were used as controls. Using these 3 esthesioneuroblastoma CT criteria, 2 blinded readers evaluating patients with esthesioneuroblastoma and controls had good diagnostic accuracy (area under the curve $=0.85$ for reader one, 0.81 for reader 2 ) for predicting esthesioneuroblastoma.
\end{abstract}

CONCLUSIONS: Esthesioneuroblastoma can present as a well-marginated intranasal lesion that unilaterally widens the olfactory recess. CT patterns can help predict esthesioneuroblastoma, potentially preventing multiple operations by instigating the correct initial operative management.

ABBREVIATIONS: ENB = esthesioneuroblastoma; OR = olfactory recess

$E^{2}$ thesioneuroblastoma (ENB) is a rare malignant neuroectodermal neoplasm arising from the neuroepithelium, most commonly within the nasal cavity olfactory recess, which makes up approximately 3\% of intranasal tumors (Fig 1). ${ }^{1,2}$ This enhancing lesion, which is most often isodense to soft tissue on CT and frequently isointense to gray matter on T2, is most commonly characterized by intracranial extension through the skull base,

Received June 9, 2017; accepted after revision September 18.

From the Departments of Radiology and Imaging Sciences (M.E.P., R.H.W., Y.A., W.F., H.R.H.), Division of Otolaryngology-Head and Neck Surgery (R.H.W., R.R.O.), and BioMedical Informatics (R.H.W.), University of Utah Health Sciences Center, Salt Lake City, Utah.

Please address correspondence to Miriam E. Peckham, MD, Departments of Radiology and Imaging Sciences, University of Utah Health Sciences Center, 30 North, 1900 East, 1A071, Salt Lake City, UT 84132-2140; e-mail: Miriam.Peckham@hsc. utah.edu; @Miriam_Peckham

三 Indicates article with supplemental on-line table.

http://dx.doi.org/10.3174/ajnr.A5464 creating a "dumbbell" shape, with the waist of the lesion located at the cribriform plate. ${ }^{3-5}$ Symptoms of this lesion are nonspecific, mirroring those of benign intranasal masses such as polyps, which can lead to delay in diagnosis, with the lesion often extending intracranially on delayed pathologic confirmation., ${ }^{4,6}$

Esthesioneuroblastoma most commonly originates in the nasal cavity olfactory recess (OR), which is a space defined by the insertion of the vertical aspect of the middle turbinate laterally, anterior skull base superiorly, and the nasal septum medially (Fig 1). ${ }^{7}$ Rare exceptions reported in the literature include lesions originating in the inferior meatus of the nasal cavity, maxillary sinus, pterygopalatine fossa, sphenoid sinus, ethmoid sinuses, and sphenoclival region. ${ }^{2,8-13}$ In contradistinction, common benign inflammatory polyps most often arise in relation to sinus outlets, most commonly in the middle or superior meatus. ${ }^{14}$ Other malignant lesions that can arise in the nasal cavity include sinonasal undifferentiated carcinoma, neuroendocrine carcinoma, and 


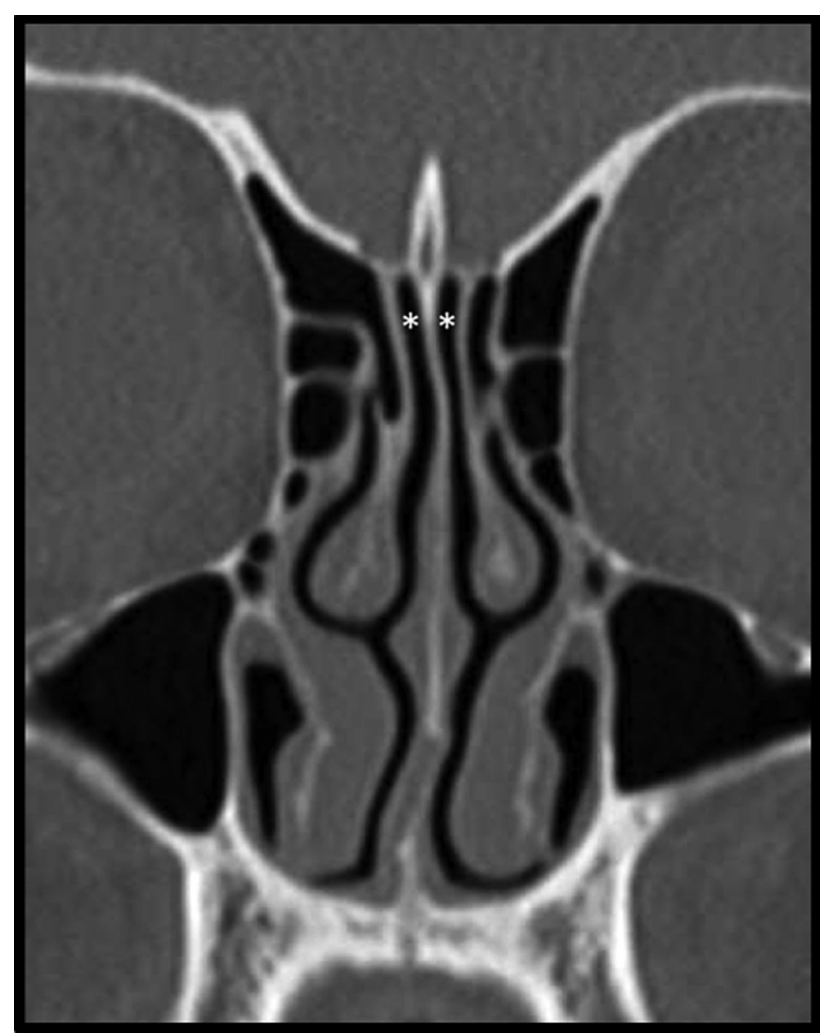

FIG 1. Coronal bone algorithm CT of the sinuses in a healthy subject demonstrates normal thin and symmetric olfactory recesses (asterisks) between the midline nasal septum and the shared superior attachments of the middle and superior turbinates laterally.

small-cell undifferentiated carcinoma, each of which can originate along the sinonasal tract. ${ }^{15}$

The most common route of spread for ENB is into the paranasal sinuses, followed by intracranial spread. These local routes of spread are most commonly staged by the Kadish staging system. ${ }^{4}$ Cervical nodal metastases have also been found up to a rate of $44 \%$, with spread most commonly seen to level II lymph nodes. ${ }^{16,17}$ Spread to retropharyngeal lymph nodes has also been reported. ${ }^{18}$ Much rarer is distant spread to the thorax and skeleton. ${ }^{19}$ Positron-emission tomography has been found helpful in evaluating nodal metastases as well as distant spread and local recurrence. ${ }^{20}$

Treatment of ENB requires complete tumor resection, usually involving anterior skull base reconstruction and primary dural closure. $^{21}$ Surgical management of ENB has more recently evolved from external-approach craniofacial resection toward an endonasal approach, which has fewer postoperative complications. ${ }^{22-24}$ An expanded endonasal approach, paired with radiation therapy and/or chemotherapy, has been found to have a high rate of local control and significantly improved survival outcomes. ${ }^{24,25}$

Small ENBs isolated to the nasal cavity can have a clinical presentation and imaging appearance like that of benign lesions such as inflammatory polyps, with smooth well-circumscribed margins rendering them indistinguishable. Location is a key differentiating factor for ENB, which characteristically originates in the olfactory recess in most cases. A prior study recommended further imaging evaluation whenever patients were found to have an opacified OR on CT; however, this study did not go into detail regarding additional $\mathrm{CT}$ findings more specific to $\mathrm{ENB}^{7} \mathrm{We}$ aimed to show that looking for additional clues, specifically osseous changes of the olfactory recess, can allow the radiologist to raise the suspicion for ENB, potentially allowing preoperative detection. Although ENB is relatively uncommon compared with other intranasal lesions, its propensity for aggressive local spread and the potential for distal metastases make it an important lesion to exclude before surgical intervention.

\section{MATERIALS AND METHODS}

The institutional review board approved this study, and it was compliant with the Health Insurance Portability and Accountability Act. Cases of extracranial ENB seen at our quaternary care center from 2009 to 2016 were identified retrospectively through a search of our picture archiving and communication system, with histologic confirmation from the institutional electronic medical record. Cases demonstrating skull base invasion or intracranial extension were excluded. The clinical presentation and imaging spectrum on preoperative CT studies were then reviewed and documented. The postoperative pathologic reports were reviewed, as well as postoperative follow-up and care. Non-ENB cases of sinus conditions involving the OR were also identified retrospectively through a search of our picture archiving and communication system. Histologic confirmation of these lesions was present in $4 / 12$ patients, with the remaining 8 showing diagnostic findings matching a specific non-ENB clinical history on initial CT, non-ENB diagnostic findings on follow-up MR imaging, or resolution of findings on subsequent imaging.

In cases of ENB, CT images were reviewed by a senior neuroradiologist with expertise in head and neck imaging and a neuroradiology fellow to characterize the location of the lesion and whether there was opacification/involvement or widening of the OR. When available, MR imaging was reviewed for the following features: T2 signal differentiation of the lesion from inflammatory disease, diffusion characteristics, enhancement characteristics, and evidence of sinus involvement. Sensitivity and specificity calculations were performed comparing these findings with histology.

Two blinded readers assessed patients with ENB and controls without ENB and, using only CT criteria, rated their level of suspicion for ENB in each case using a standard rating scale from 1 to $5(1=$ definitely not, $2=$ probably not, $3=$ equivocal, $4=$ probably yes, $5=$ definitely yes). Receiver operating characteristic curves were generated for individual readers to evaluate diagnostic accuracy. Statistical analyses were performed using commercial statistical analysis software (STATA, Release 14; StataCorp, College Station, Texas).

\section{RESULTS}

Thirty cases of possible ENB on imaging were reported at our quaternary care institution from 2009 to 2016 . Of these cases, 20 were histology-proved ENB, and the 10 remaining cases had varying diagnoses, including inflammatory polyp, squamous cell carcinoma, metastatic disease, sinonasal undifferentiated carcinoma, and epithelial adenomatoid hamartoma. Of the 20 pathologically proved cases of ENB, 12 showed intracranial extension and/or imaging evidence of skull base invasion and were therefore excluded from this study. Eight cases (3 women) were completely 


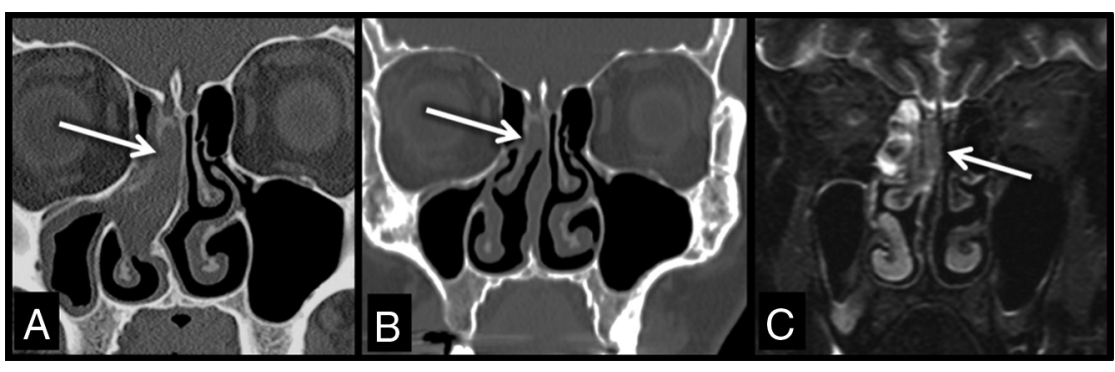

FIG 2. Coronal bone algorithm $C T(A$ and $B)$ and coronal T2-weighted MR image $(C)$ obtained prepolypectomy $(A)$, and postpolypectomy $(B$ and $C$ ) demonstrate a soft-tissue-density lesion (arrows) that extends to the cribriform plate and widens the right olfactory recess. Opacification of the paranasal sinuses cannot be differentiated from the lesion on CT; however, paranasal sinus T2 bright secretions are readily discerned on MR imaging from the lesion (arrow), which is isointense to gray matter $(C)$. This patient required an additional anterior skull base resection once ENB was diagnosed after routine polypectomy.

extracranial, showing no evidence of skull base invasion, with an age range of 40-74 years (mean age, 58 years). Of these extracranial cases, imaging and histologic studies demonstrated that 3 of the lesions were completely isolated to the nasal cavity and 5 were intranasal with variable adjacent sinus involvement (On-line Table). Initial CT scans were available for review in all cases. MR imaging was available for 5 patients, in 2 performed preoperatively, in 2 performed after initial polypectomy, and in 1 performed after recurrence (On-line Table).

Twelve non-ENB cases of sinus conditions (7 women) with OR involvement between 2009 and 2017 were obtained with an age range of 16-81 years (mean age, 48 years). These cases had varying diagnoses including CSF leak, encephalocele, allergic rhinitis, chronic sinusitis, antrochoanal/inflammatory polyp, mycetoma, and glomus tumor.

\section{Clinical Features of Intranasal Esthesioneuroblastoma}

Difficulty breathing due to nasal stuffiness and fullness was the most common presenting symptom in patients with intranasal ENB. Other symptoms included anosmia and rhinorrhea. Five patients had presurgical biopsies before resection. Three patients required an additional operation after referral to our institution for skull base resection after ENB was found incidentally during routine nasal polypectomy. On presentation, there was no clinical or imaging evidence in these patients of nodal involvement.

\section{CT Features of Intranasal Esthesioneuroblastoma}

All cases had CT demonstrating a circumscribed intranasal polypoid lesion with the following features: 1 ) its epicenter in a unilateral OR, 2) causing asymmetric osseous remodeling/widening of the OR, and 3) extending to the cribriform plate. All except 1 patient had additional opacification of several paranasal sinuses; however, extension versus fluid entrapment could not be discerned by CT (Fig 2).

Six original preoperative CT reports were available. In 2 cases, the radiologist suspected a malignant lesion and recommended follow-up imaging. The 4 remaining cases had been referred from outside facilities and the patients had already undergone biopsy; however, the biopsy results appeared to be available to the radiologist in only half of those patients because the chance of malignancy was dismissed in 2 patients and, in the remaining 2 patients, the results were described as consistent with prior ENB biopsy. The preoperative imaging diagnosis of ENB was never specifically stated as a possibility in any cases without prior biopsy.

\section{MR Imaging Features of Intranasal Esthesioneuroblastoma}

In the 5 cases with MR imaging, all lesions were $\mathrm{T} 2$ isointense to gray matter and showed diffuse enhancement to a lesser degree than the adjacent nasal mucosa. When available (3 subjects), diffusion-weighted imaging demonstrated hyperintense DWI signal intensity and hypointense ADC signal intensity within the mass. Entrapped fluid within the paranasal sinuses was differentiated from tumor extension by its $\mathrm{T} 2$ hyperintensity, greater than the intrinsic $\mathrm{T} 2$ signal within the ENB mass (Fig 2C). All MR imaging cases that had imaging evidence of paranasal sinus tumor extension (3 subjects) were confirmed histologically.

\section{Histologic Findings}

Following surgical resection, 3 of these cases were proved histologically to be isolated to the nasal cavity, while the 5 remaining cases showed additional sinus involvement. Dural margins were negative in $7 / 8$ subjects, with this information not available in 1 subject.

Histologic grading of these lesions showed 1 case with grade 1 , three cases with grade 2 , and 2 cases with grade 3 morphology. These grades did not correspond with sinus involvement. Two patients with grade 3 lesions had local recurrence, with 1 of the patients also showing nodal recurrence 3 years after the initial resection. No recurrence was found in the patients with grade 1 or 2 lesions (On-line Table).

\section{Comparison with Controls}

Among the 12 patients without ENB with sinus conditions involving the OR, only $3 / 12$, all of which were masses (glomus tumor, inflammatory polyp, and epithelial adenomatoid hamartoma), showed unilateral and asymmetric widening/remodeling of the OR. The remaining 9 cases consisted mostly of non-mass-like infectious/inflammatory processes that opacified but did not asymmetrically widen/remodel the OR (Fig 3). The 3 CT criteria of a mass with its epicenter in the OR, causing asymmetric osseous widening/remodeling of the $\mathrm{OR}$, and extending to the cribriform plate, were demonstrated in all ENB cases (sensitivity of $100 \%$ compared with histology) and in 3 cases without ENB (specificity of $75 \%$ compared with histology).

\section{Performance of Blinded Readers}

Blinded readers demonstrated strong individual accuracy in predicting ENB using the $3 \mathrm{CT}$ criteria, with reader 1 having an area under the curve of $0.85 \pm 0.087$ and reader 2 having an area under the curve of $0.81 \pm 0.089$ (Fig 4 ). When results were dichotomized between ENB absence and presence, the sensitivity for reader 1 was $87.5 \%$ with a specificity of $58.3 \%$ and sensitivity for reader 2 was $50.0 \%$ with a specificity of $83.3 \%$. 


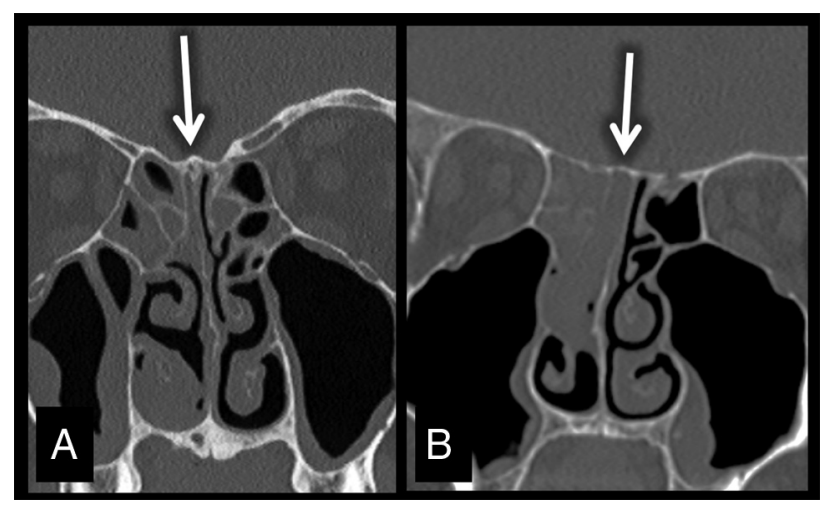

FIG 3. Coronal bone algorithm $C T(A$ and $B)$ images obtained in a control subject with chronic sinusitis $(A)$ and a subject with ENB $(B)$. While there is asymmetric opacification of the right OR with extension to the cribriform plate in the subject with chronic sinusitis $(A)$, the $\mathrm{OR}$ is not widened. This finding contrasts with that in the subject with ENB who demonstrates additional asymmetric widening/remodeling of the OR. Both subjects have opacification of the adjacent paranasal sinuses, which, in the subject with ENB, was found to represent tumor extension. The subject with ENB underwent additional anterior skull base resection surgery when ENB was found after routine polypectomy.

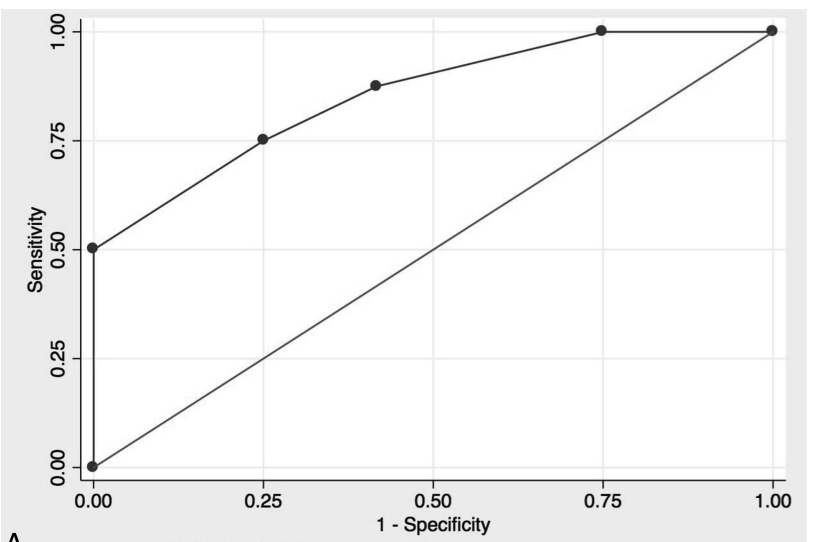

A

Area under ROC curve $=0.854$

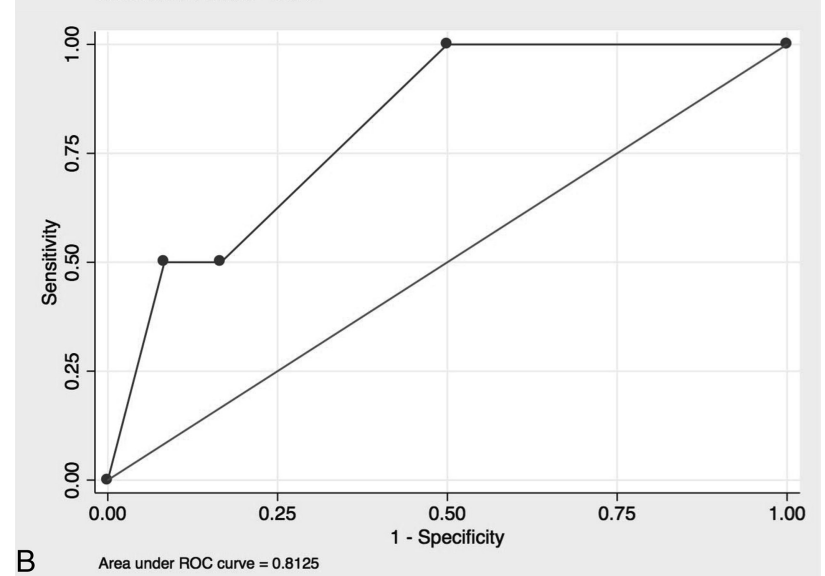

FIG 4. Receiver operating characteristic curves for reader $1(A)$ and reader $2(B)$. Sensitivity and specificity varied between both readers; however, the overall diagnostic accuracy was strong, with reader 1 showing an area under the curve of 0.85 , and reader two, 0.81 .

\section{DISCUSSION}

Esthesioneuroblastoma is a rare neuroendocrine mass that, when isolated to the nasal cavity, can appear very similar to benign sinonasal masses on CT. Because this lesion is malignant and requires a more invasive operation for resection than a standard polypectomy, patients with intranasal lesions without intracranial involvement were studied to define distinguishing imaging characteristics. On the basis of these characteristics, CT imaging findings are established to recommend further work-up with MR imaging and/or biopsy before complete surgical resection.

Patients presenting with intranasal ENB were of a similar age range and had presenting symptoms of nasal stuffiness and fullness like those seen with benign entities. Reflecting this nonspecific presentation, malignancy was not initially suspected in almost half of the patients and a standard nasal polypectomy was performed without obtaining a presurgical biopsy or performing further imaging. This resulted in the necessity to perform a second more extensive operation for anterior skull base resection when postoperative pathology results showed ENB.

The margins of the intranasal ENB mass did not significantly differ from those in benign inflammatory lesions, with both entities appearing smooth and well-circumscribed with homogeneous soft-tissue density. Although these findings were overall similar, the 3 characteristics of a mass epicenter in a unilateral OR causing widening/osseous remodeling and extending to the cribriform plate showed high sensitivity and moderate specificity for ENB. Of the 9 controls with nonmass lesions opacifying the OR, none demonstrated all 3 of these characteristics. These 3 characteristics had strong diagnostic accuracy when used by blinded readers to identify ENB.

A consistent finding in patients with ENB was adjacent paranasal sinus opacification, which was seen in 7/8 patients, representing tumor in 5 cases. The sinus opacification in the 2 patients with isolated intranasal ENB was found to represent entrapped fluid related to sinus blockage from the intranasal mass, a finding that was readily discernible on MR imaging but not on CT. Because fluid and tumor within the paranasal sinuses had similar density on CT, that sequence alone was not reliable for evaluating local disease spread. These cases demonstrated that the presence of an intranasal mass can create a confusing picture of paranasal sinus opacification and that the $3 \mathrm{CT}$ criteria regarding the $\mathrm{OR}$ were the most important imaging findings for predicting ENB.

The T2-weighted and STIR MR imaging sequences were readily able to differentiate tumor from paranasal sinus inflammatory disease. The coronal plane was the most helpful because it clearly showed involvement and widening of the OR and extension of the lesions to the cribriform plate. The T2 sequence aided differentiation of the ENB mass from other common benign polyps, with ENB lesions demonstrating T2 isointensity and hypointense ADC signal, both of which correlate with increased cellularity. ${ }^{5}$ The MR imaging T2-weighted sequences were also helpful for evaluating local disease spread into the paranasal sinuses because hyperintense entrapped paranasal sinus fluid could be readily differentiated from the more T2 isointense signal of the ENB tumor. The ability to differentiate ENB from inflammatory entities by signal characteristics further supports the importance of obtaining preoperative MR imaging when ENB is suspected by CT.

The importance of prompting further work-up when there is OR opacification and widening on CT is demonstrated because 
almost half of the patients with intranasal ENB required a second more extensive operation for complete resection. Two cases that necessitated repeat surgery had outside radiology reports that were not available for review; however, this lesion was clearly not initially suspected by the managing physicians in reviewing the electronic medical record. Most interesting, in 2 cases in which a presurgical biopsy was performed at an outside institution, the radiologist apparently did not have access to these results and therefore did not suspect or prompt any further work-up for malignancy based on the original CT findings. Also, ENB was never specifically mentioned as a possibility in any of the CT reports in which biopsy results were unavailable. These results strengthen the concept that in CT sinus studies, evaluation for opacification/ widening of the OR must be included on the imaging checklist.

Management of ENB requires complete surgical resection with clear margins whenever anatomically feasible. Adjuvant therapy is often necessary, due to higher grade lesions or close/positive margins. Extracranial ENB is almost always amenable to complete surgical resection, due to rare involvement with the orbits and lack of cerebral involvement. Because the tumor arises from nasal olfactory epithelium near the skull base, resection of the dura as a superior margin is nearly always necessary. This procedure is readily performed endonasally in a number of skull base centers, without the need for a formal craniotomy. In a rare lesion with a radiographically clear area between the lesion and the skull base, the surgeon may choose to spare the dura. Knowledge of the histology of olfactory lesions is critical because the management of an ENB differs considerably from that of a benign neoplasm and from inflammatory polyps. Benign lesions typically do not require skull base/dural resection and inflammatory polyps often are treated even less aggressively, with surgery focused on improving access for topical anti-inflammatory therapy. ${ }^{26}$ With the otolaryngologist having the correct diagnosis of an extracranial ENB, the correct definitive operation is performed initially and the patient is spared multiple trips to the operating room.

The greatest limitations in this case-control study were low power because of the rarity of extracranial ENB, as well as the subtle imaging changes associated with extracranial ENB in comparison with cases without ENB involving the OR, which made blinded reads challenging. However, even with the limited number of cases and subtle imaging characteristics, blinded readers demonstrated strong individual accuracy for predicting ENB using this method, with reader 1 demonstrating high sensitivity and reader 2 demonstrating high specificity. Although extracranial ENB is rare, the consistency of its CT imaging findings does provide helpful information for guiding care.

\section{CONCLUSIONS}

Esthesioneuroblastoma can present as a well-marginated polypoid mass lesion isolated to the nasal cavity. Because this lesion is managed differently from more common benign nasal entities, this important diagnosis should be included in the differential whenever a mass follows 3 important CT criteria: epicenter within a unilateral OR, widening/osseous remodeling of the OR, and extension of the lesion to the cribriform plate. These criteria were sensitive for ENB and provided strong diagnostic accuracy when used by blinded readers. The presence of these findings should elicit preoperative MR imaging and/or biopsy, which can aid the surgeon in operative planning and prevent routine nasal polypectomy for resection of these aggressive lesions.

\section{REFERENCES}

1. Berger L, Luc R. L'esthesioneuroepitheliome olfactif. Bull Assoc Fr Etude Cancer 1924;13:410-21

2. Lee JY, Kim HK. Primary olfactory neuroblastoma originating from the inferior meatus of the nasal cavity. Am J Otolaryngol 2007; 28:196-200 CrossRef Medline

3. Yu T, Xu YK, Li L, et al. Esthesioneuroblastoma methods of intracranial extension: CT and MR imaging findings. Neuroradiology 2009;51:841-50 CrossRef Medline

4. Dias FL, Sa GM, Lima RA, et al. Patterns of failure and outcome in esthesioneuroblastoma. Arch Otolaryngol Head Neck Surg 2003;129: 1186-92 CrossRef Medline

5. Dublin AB, Bobinski M. Imaging characteristics of olfactory neuroblastoma (esthesioneuroblastoma). J Neurol Surg B Skull Base 2016; 77:1-5 CrossRef Medline

6. London NR Jr, Reh DD. Differential diagnosis of chronic rhinosinusitis with nasal polyps. Adv Otorhinolaryngol 2016;79:1-12 CrossRef Medline

7. Hoxworth JM, Glastonbury CM, Fischbein NJ, et al. Focal opacification of the olfactory recess on sinus CT: just an incidental finding? AJNR Am J Neuroradiol 2008;29:895-97 CrossRef Medline

8. Jiang ZY, Katz A, Francis C. Hyponatremia and right maxillary sinus mass. JAMA Otolaryngol Head Neck Surg 2015;141:1021-22 CrossRef Medline

9. Unal A, Ozlugedik S, Tezer MS, et al. An atypical esthesioneuroblastoma of the inferior nasal cavity and maxillary sinus: report of a case. Tumori 2006;92:440-43 Medline

10. Matsunaga M, Nakagawa T, Sakamoto T, et al. Sphenoid esthesioneuroblastoma arising from the hindmost olfactory filament. Auris Nasus Larynx 2015;42:170-72 CrossRef Medline

11. Leon-Soriano E, Alfonso C, Yebenes L, et al. Bilateral synchronous ectopic ethmoid sinus olfactory neuroblastoma: a case report. Am J Case Rep 2016;17:268-73 CrossRef Medline

12. Seccia V, Lenzi R, Casani AP, et al. Ectopic olfactory neuroblastoma arising in the pterygopalatine fossa. Otolaryngol Head Neck Surg 2010;142:460-61 CrossRef Medline

13. Purohit B, Winder T, Maggio EM, et al. Aggressive primary olfactory neuroblastoma of the sphenoclival region: a case report and literature review. Laryngoscope 2015;125:822-25 CrossRef Medline

14. Larsen PL, Tos M. Origin of nasal polyps: an endoscopic autopsy study. Laryngoscope 2004;114:710-19 CrossRef Medline

15. Hofer MJ, Rohlfs J, Teymoortash A, et al. A 62-year-old female with an intranasal mass extending into the lamina cribrosa. Brain Pathol 2013;23:105-08 CrossRef Medline

16. Rinaldo R, Ferlito A, Shaha AR, et al. Esthesioneuroblastoma and cervical lymph node metastases: clinical and therapeutic implications. Acta Otolaryngol 2002;122:215-21 CrossRef Medline

17. Howell MC, Branstetter BF 4th, Snyderman CH. Patterns of regional spread for esthesioneuroblastoma. AJNR Am J Neuroradiol 2011;32: 929-33 CrossRef Medline

18. Zollinger LV, Wiggins RH, Cornelius RS, et al. Retropharyngeal lymph node metastasis from esthesioneuroblastoma: a review of the therapeutic and prognostic implications. AJNR Am J Neuroradiol 2008;29:1561-63 CrossRef Medline

19. Pickuth D, Heywang-Kobrunner S, Speilman RP. Computed tomography and magnetic resonance imaging features of olfactory neuroblastoma: an analysis of 22 cases. Clin Otolaryngol Allied Sci 1999;24:457-61 CrossRef Medline

20. Broski SM, Hunt CH, Johnson GB, et al. The added value of $18 \mathrm{~F}$ FDG PET/CT for evaluation of patients with esthesioneuroblastoma. J Nucl Med 2012;53:1200-06 CrossRef Medline

21. Tomio R, Toda M, Tomita T, et al. Primary dural closure and anterior cranial base reconstruction using pericranial and nasoseptal 
multi-layered flaps in endoscopic-assisted skull base surgery. Acta Neurochir (Wien) 2014;156:1911-15 CrossRef Medline

22. Lund VJ, Howard D, Wei W, et al. Olfactory neuroblastoma: past, present, and future? Laryngoscope 2003;113:502-07 CrossRef Medline

23. Smee RI, Broadley K, Williams JR, et al. Retained role of surgery for olfactory neuroblastoma. Head Neck 2011;33:1486-92 CrossRef Medline

24. Hwang CS, Seo YW, Park SC, et al. Role of surgical treatment for esthesioneuroblastomas: 31-year experience at a single institution. J Craniomaxillofac Surg 2017;45:120-26 CrossRef Medline

25. Roxbury CR, Ishii M, Gallia GL, et al. Endoscopic management of esthesioneuroblastoma. Otolaryngol Clin North Am 2016;49:153-65 CrossRef Medline

26. Orlandi RR, Kingdom TT, Hwang PH, et al. International Consensus Statement on Allergy and Rhinology: Rhinosinusitis. Int Forum Allery Rhinol 2016; (suppl 1):S22-209 CrossRef Medline 